\title{
Selective Histamine Uptake Rescues Photo- and Mechanoreceptor Function of Histidine Decarboxylase-Deficient Drosophila Mutant
}

\author{
Jörg Melzig, ${ }^{1}$ Martin Burg, ${ }^{2}$ Matthias Gruhn, ${ }^{1}$ William L. Pak, ${ }^{2}$ and Erich Buchner ${ }^{1}$ \\ 1Theodor-Boveri Institut für Biowissenschaften, Lehrstuhl für Genetik, Universität Würzburg, D-97074 Würzburg, \\ Germany, and 2Department of Biological Sciences, Purdue University, West Lafayette, Indiana 47907
}

In insects, histamine is found both in the peripheral nervous system (PNS) and in the CNS and is known to function as a fast neurotransmitter in photoreceptors that have been shown to express selectively the hdc gene. This gene codes for histidine decarboxylase (HDC), the enzyme for histamine synthesis. Fast neurotransmission requires the efficient removal of the transmitter from the synaptic cleft. Here we identify in Drosophila photo- and mechanoreceptors a histamine uptake mechanism that can restore the function of these receptors in mutants unable to synthesize histamine. When apparent null mutants for the hdc gene imbibe aqueous histamine solution or are genetically "rescued" by a transgene ubiquitously expressing histidine decarboxylase under heat-shock control, sufficient amounts of histamine selectively accumulate in photo- and mechanoreceptors to generate near-normal electrical responses in second-order visual interneurons and qualitatively to restore wild-type visual and mechanosensory behavior. This strongly supports the proposal that histamine functions as a fast neurotransmitter also in a certain class of mechanoreceptors. A set of CNS-intrinsic neurons that in the wild type contain high concentrations of histamine apparently lacks this uptake mechanism. We therefore speculate that histamine of intrinsic neurons may function as a neuromodulator rather than as a fast transmitter.

Key words: histamine; uptake; neurotransmission; neuromodulation; vision; mechanosensation; behavior; insect; Drosophila
In the adult acalypteran fly Drosophila, histamine is found in all photoreceptors, in mechanosensory neurons of hair sensilla on the entire body surface, and in $\sim 42$ neurons intrinsic to the CNS (Pollack and Hofbauer, 1991; Nässel and Elekes, 1992; Buchner et al., 1993). Histamine-gated chloride channels have been identified and characterized in the first-order visual interneurons of various dipteran flies and are presumed to mediate synaptic transmission from photoreceptors to these cells (Hardie, 1989; Skingsley et al., 1995). Electrophysiological and behavioral analyses of mutant flies defective in the $h d c$ gene, which codes for the histamine-synthesizing enzyme histidine decarboxylase, have demonstrated that in the photoreceptors of the compound eyes and, presumably, the mechanosensory neurons of cuticular bristles, histamine functions as the major or sole neurotransmitter. Flies homozygous for the apparent null allele $h d c^{J K 910}$ lack electroretinogram components indicative of photoreceptor synaptic transmission, correspondingly are blind, and show severe defects in certain mechanosensory behavior such as grooming or bristle scratch reflexes (Burg et al., 1993; Melzig et al., 1996). No information is as yet available on the role of histamine in the intrinsic cells. For a substance to function as a fast transmitter, as would be required for neurons mediating fast visual and tactile reflexes, it must not only act directly on postsynaptic ion channel receptors but also has to be removed effectively from the synaptic cleft.

\footnotetext{
Received Feb. 5, 1998; revised June 5, 1998; accepted July 6, 1998.

This work was funded by Deutsche Forschungsgemeinschaft Grant Bu566 to E.B. We would like to thank M. Heisenberg, A. Hofbauer, and C.-F. Wu for valuable discussions; S. Buchner, D. Reisch, and R. Wolf for preparing the figures; and D. Dudaczek for excellent technical help.

Correspondence should be addressed to Dr. Erich Buchner, Theodor-Boveri Institut für Biowissenschaften, Lehrstuhl für Genetik, Am Hubland, D-97074 Würzburg, Germany.

Copyright (ㄷ) 1998 Society for Neuroscience $\quad 0270-6474 / 98 / 187160-07 \$ 05.00 / 0$
}

Because amine metabolic breakdown is rather slow, one would expect removal to be achieved by selective high-affinity uptake of the transmitter. It was therefore puzzling that no selective accumulation of histamine was observed when Drosophila heads were incubated in a solution containing $20 \mu \mathrm{M}\left[{ }^{3} \mathrm{H}\right]$ histamine (Sarthy, 1991). However, an activity-dependent uptake mechanism for histamine has recently been discovered in barnacle photoreceptors (Stuart et al., 1996). Here we demonstrate for Drosophila that food-supplied or ubiquitously synthesized histamine is selectively and efficiently taken up in vivo into photo- and mechanoreceptors but not into CNS neurons of mutants lacking the selectively expressed $h d c$ gene. The function of visual and mechanosensory pathways in these histamine-supplemented mutants is primarily restored.

\section{MATERIALS AND METHODS}

Flies

Wild-type strains Berlin-K, Oregon-R, and Canton-S were used indiscriminately because no differences were seen. The $h d c$ mutant $h d c^{J K 910}$ was kindly provided by Dr. J. Merriam (University of California, Los Angeles), and the alleles $h d c^{P 211}, h d c^{P 217}$, and $h d c^{P 218}$ were generated in the laboratory of W.L.P. In the behavioral experiments, only flies not older than 1 week were used.

\section{Transformation rescue of $\mathrm{hdc}^{\mathrm{JK} 910}$ mutant}

The $h d c^{J K 910}$ mutant allele had not been tested in the previous rescue experiments by transient expression of the $h d c$ cDNA (Burg et al., 1993). $h d c^{J K 910}$ mutants bearing a single copy of the pCaSpeR-hs- $h d c$ rescue vector were heat shocked as described below.

\section{Application of histamine}

All stocks were maintained at $18^{\circ} \mathrm{C}$ in a $12 \mathrm{hr}$ light/dark cycle on standard medium. Flies were transferred to a vial containing a Whatman filter soaked either in distilled water (control) or in an aqueous 5\% histaminediphosphate (Sigma, St. Louis, MO) solution and kept there for $3 \mathrm{hr}$ 
before the histochemical experiments and overnight before the behavioral and electrophysiological experiments. For immunohistochemistry, sections from histamine-fed and control flies, or from heat-shocked and nonheat-treated transformants, respectively, were processed together on the same microscope slide to ensure identical staining conditions.

\section{Immunohistochemistry}

Flies were fixed for $2.5 \mathrm{hr}$ in an ice-cold solution containing 4\% 1-ethyl3-(3-dimethylaminopropyl)carbodi-imide (Sigma) in $67 \mathrm{~mm}$ phosphate buffer, pH 7.4, washed overnight in $25 \%$ sucrose in PBS (130 mM NaCl, $7 \mathrm{mM} \mathrm{Na}_{2} \mathrm{HPO}_{4}$, and $3 \mathrm{mM} \mathrm{NaH} \mathrm{PO}_{4}, \mathrm{pH} \mathrm{7.4),} \mathrm{embedded} \mathrm{in} 16 \%$ carboxymethyl-cellulose, shock frozen in melting nitrogen, and sectioned at $10 \mu \mathrm{m}$ thickness on a cryostat microtome. The sections were incubated overnight with an antiserum directed against histamine (dilution 1:1000 in PBS; PAN19C; Incstar, Stillwater, MA) and stained with the peroxidase-antiperoxidase technique as described previously (Buchner et al., 1993).

\section{Heat-shock protocol}

The vector used for rescue of the $h d c$ mutant phenotype, expressing the $h d c$ cDNA under hsp70 control, has been described previously (Burg et al., 1993). All transformants were crossed into the $h d c^{J K 910}$ mutant background, which has been shown not to synthesize any immunohistochemically detectable amounts of histamine (Melzig et al., 1996). Transformants of specific ages (2-10-d-old) were heat shocked for $30 \mathrm{~min}$ at $37^{\circ} \mathrm{C}$. Longer heat shocks were not attempted, because the heat treatment described resulted in clear immunohistochemical staining and a normal electroretinogram (ERG), both of which was observed for at least 5 weeks after a single heat pulse. All flies were cultured at $19^{\circ} \mathrm{C}$ before and after heat treatments, because higher temperatures induced expression from the $\mathrm{pCaSpeR}-\mathrm{hs}-h d c$ vector. Heat-shocked flies were analyzed either for ERG or immunohistochemical rescue of the mutant phenotype at least $1 \mathrm{~d}$ later. From five individual transformant lines obtained, one was selected for these studies because it exhibited the least amount of constitutive expression of the $h d c$ cDNA under noninduced conditions. All other lines demonstrated similar results, although background staining was higher.

\section{ERGS}

Three-day-old flies were attached to a holder by dental cement (ESPE, Seefeld, Germany) that was also used to immobilize the head and legs. Borosilicate glass microelectrodes (Clark) were filled with Drosophila Ringer's solution (200 $\mathrm{mm} \mathrm{NaCl}, 2 \mathrm{mM} \mathrm{CaCl}_{2}$, and $5 \mathrm{~mm} \mathrm{KCl}$ ) and placed in the thorax (indifferent electrode) or just beneath the cornea in the center of the eye (recording electrode; tip resistance, $\sim 10-15 \mathrm{M} \Omega$ ). Recordings were made at $\sim 18.5^{\circ} \mathrm{C}$ after a minimum of 20 min of dark adaptation under dim red light. In the feeding experiments, light flashes of $2800 \mathrm{~lx}$ and $0.8 \mathrm{sec}$ duration at $1.2 \mathrm{sec}$ intervals were given from a clear green light-emitting diode [Telefunken TLHG; $\lambda, 565 \mathrm{~nm}$ ] placed $\sim 0.5$ $\mathrm{cm}$ in front of the eye (Gruhn, 1995). For stimulation of transgenic animals, a 100-fold-attenuated tungsten light source was focused onto the eye as described previously (Burg et al., 1993).

\section{Behavioral paradigms}

"Buridan's paradigm." In this behavioral test, flies with clipped wings walk freely on a circular platform surrounded by a water-filled moat (Götz, 1980). When the platform is placed in an illuminated arena containing two vertical black stripes at opposite positions outside the moat, wild-type flies will keep running back and forth between the two stripes. Their tracks are recorded by a video camera and plotted by a computer. Tracks of defined time periods indicate by their lengths the internal predisposition for locomotion and by their systematic deviation from rotational symmetry the ability of the fly to perceive visually the black stripes. These experiments were performed with at least 10 animals from each group.

Bristle reflexes. We used a modified test for stimulation of thoracic bristles (Vandervorst and Ghysen, 1980) that is described in detail elsewhere (Melzig et al., 1996). In brief, thoracic or interommatidial bristles of tethered flies were touched gently with an eyelash attached to a hand-held rod. A total of 10 stimulations were given at 1 min intervals. A response was rated positive if the stimulus induced a clear movement of the legs, either scratching the body surface or attempting to escape.

Grooming behavior. Again, details on the quantitative grooming assay used have been described (Melzig et al., 1996). Briefly, 10 flies (five males and five females, to keep body surface constant) were anesthetized on ice and coated in a $4^{\circ} \mathrm{C}$ room with Orange $G$ dust (Merck, Darmstadt, Germany) by shaking them for $20 \mathrm{sec}$ in a Plexiglas vial containing 10-50 $\mathrm{mg}$ of dust. Excess dust was removed by shaking the flies for $5 \mathrm{sec}$ on a sieve (mesh size, $1 \mathrm{~mm}$ ). After transfer to an empty culture vial in an $18^{\circ} \mathrm{C}$ room, the flies were left undisturbed for defined times (i.e., $10 \mathrm{~min}$ to recover and $0,30,120,180$, or 240 min to groom and remove the dust). After these periods, flies were killed by freezing at $-80^{\circ} \mathrm{C}$, transferred to a $250 \mathrm{ml}$ beaker, and washed for $2 \mathrm{~min}$ in a defined volume of water, and the absorption of this wash was measured in a spectrophotometer (Beckman DU-40; $\lambda, 480 \mathrm{~nm})$.

\section{RESULTS}

\section{Immunohistochemical experiments}

The distribution of histamine in the wild-type CNS of adult Drosophila is shown schematically in Figure 1. For clarity we have drawn separately in the left hemisphere the histaminergic projections from the peripheral nervous system (terminals of photoreceptors and mechanosensory receptor neurons of cuticular bristles) and in the right hemisphere the histamine-containing CNSintrinsic neurons with their arborizations. Figure 2 shows immunohistochemical stainings of horizontal sections of wild type $(a)$, untreated $h d c^{J K 910}$ mutants $(b, e, g)$, mutants fed on histamine-diphosphate solution $(c, f, h)$, and heat-treated mutants containing an $h d c$ transgene under the control of a heat-shock promoter $(d)$. Although all specific staining is missing in the untreated mutants (Melzig et al., 1996), sections from histaminefed mutants demonstrate selective uptake into the photoreceptor terminals R1-6 and R7/8 (Fig. 2c, long arrows) as well as into sensory neurons of cuticular bristles. The latter is exemplified here by the staining of axons in the antennal nerve (Fig. $2 c$, short arrow), of the base of a thoracic macrochaeta (Fig. $2 f$, arrow), and of axons and terminals of the wing nerve, the leg nerves, and the abdominal nerves (Fig. $2 h$, arrows). No uptake is observed into perikarya, axons, or terminals of those CNS-intrinsic neurons of the brain (Fig. 2a, arrowheads) or the thoracoabdominal ganglia that in the wild type contain histamine (cf. also Pollack and Hofbauer, 1991; Buchner et al., 1993). As a control, we subjected wild-type flies to the same feeding conditions and observed, on increased background, a small but significant decrease in staining intensity of the photoreceptor terminals, compared with that of the water-fed wild type (data not shown). Several explanations can be proposed for this unexpected finding (see Discussion). No information is at present available on the biological half-life of histamine in the mutant tissue after feeding or on the duration of the associated functional "rescue."

Similar results were obtained with transgenic $h d c^{J K 910}$ mutants capable of synthesizing histamine because of ubiquitously expressed histidine decarboxylase (HDC). Although several flies bearing the pCaSpeR-hs- $h d c$ construct did demonstrate some low levels of histamine staining in photoreceptors without heat shock, the amount of histamine detected in these cells after the transformants had been heat shocked was close to wild-type levels in many cases (Fig. $2 d ; n=23$ ). In 16 of these preparations, histamine was also detected in the antennal nerve or other mechanosensory cells. As in the feeding experiments, no histamine staining beyond background levels was ever observed in the CNS-intrinsic neurons that in the wild type contain histamine. Thus selective histamine accumulation in these cells critically depends on selective HDC expression and cannot be achieved by histamine uptake. On the other hand, sections obtained from flies as long as 5 weeks after a single heat shock clearly demonstrated that high levels of histamine in photoreceptor terminals are maintained even long after heat-induced HDC expression 


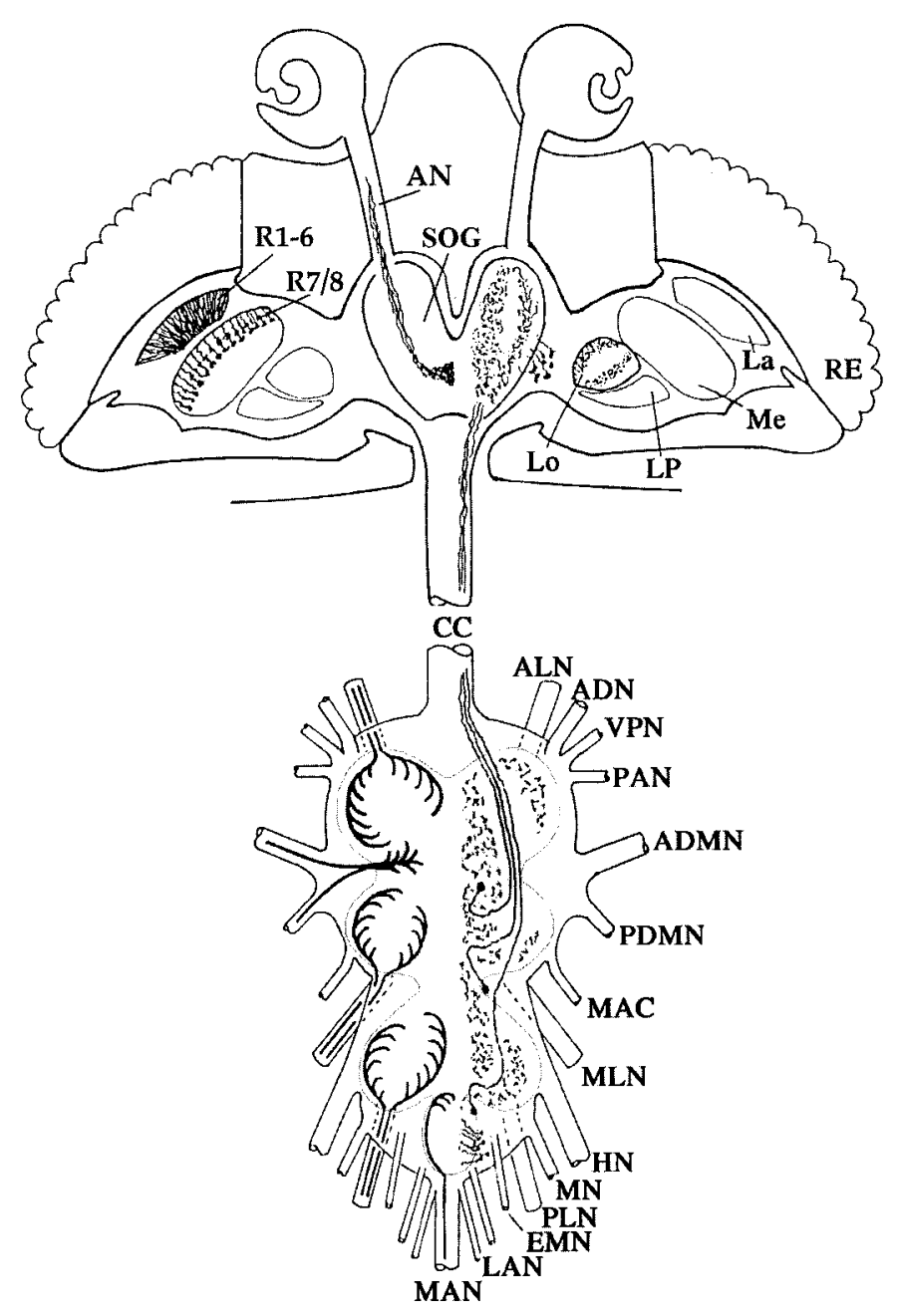

Figure 1. Histamine distribution in the Drosophila imaginal nervous system. The schematic drawing of head and thoracoabdominal ganglia of Drosophila illustrates histaminergic axons and terminals derived from photoreceptors and bristle mechanoreceptors of the peripheral nervous system (left hemisphere) and the histamine-containing intrinsic neurons of the CNS with their arborizations (right hemisphere). $A D N$, Anterior dorsal nerve; $A D M N$, anterior dorsal mesothoracic nerve; $A L N$, prothoracic leg nerve; $A N$, antennal nerve; $C C$, cervical connective; $E M N$, extra metathoracic nerve; $H N$, haltere nerve; $L a$, lamina; $L A N$, first and second lateral abdominal nerves; $L o$, lobula; $L P$, lobula plate; $M A C$, metathoracic accessory nerve; $M A N$, medial abdominal nerve; $M e$, medulla; $M L N$, mesothoracic leg nerve; $M N$, metathoracic nerve; $P A N$, prothoracic accessory nerve; $P D M N$, posterior dorsal mesothoracic nerve; $P L N$, metathoracic leg nerve; $R 1-8$, retinula cells R1-8; $R E$, retina; $S O G$, subesophageal ganglion; $V P N$, ventral prosternal nerve.

ceased. It is not known, however, whether this phenomenon is caused by low histamine turnover, high HDC stability, or basal $\mathrm{HDC}$ expression from the transgene at $19^{\circ} \mathrm{C}$.

\section{ERGs}

The summed ERG potential that can be recorded from the corneal surface of the compound eyes of Drosophila consists of two major components. The receptor component derives from the membrane currents of the photoreceptors and has a waveform that is essentially the inverse of the receptor potential recorded intracellularly from single photoreceptor cells. The on- and offtransients (Fig. 3a, arrowheads), on the other hand, are known to be produced in the lamina, the first visual neuropil, and reflect the signals generated in the large monopolar cells (LMCs) of the lamina. Thus these transients depend on, and are indicative of, intact synaptic transmission from photoreceptors to LMCs (Heisenberg, 1971; Pak, 1975; Coombe, 1986).

ERGs from wild-type flies, $h d c^{J K 910}$ mutants, histamine-fed mutants, and heat-shocked transformants are shown in Figure 3. $h d c^{J K 910}$ mutants without an exogenous or transgenic histamine supply display neither on- nor off-transients (Fig. 3b), as had been reported previously (Burg et al., 1993). After histamine feeding of the $h d c^{J K 910}$ flies, the ERG transients essentially recover (Fig. $3 c$, left; $n=4)$. Siblings of the transgenic flies investigated by histamine immunohistochemistry were also subjected to the ERG analysis. The data $(n>25)$ demonstrated that transient expression of the $h d c$ cDNA clearly rescues the ERG phenotype of $h d c^{J K 910}$ (Fig. 3c, right) in the same manner that the feeding of histamine rescued the phenotype. This rescue, observed within 1 hr of the end of heat shock, appears to be permanent; transformant flies that were heat shocked and then aged for an additional 6 weeks at $19^{\circ} \mathrm{C}$ still demonstrated on- and off-transients in the ERG, unlike nonheat-shocked transformants of similar age that were raised at $19^{\circ} \mathrm{C}(n>25$; data not shown). Thus histamine in the food or synthesized by ubiquitously expressed HDC can restore synaptic transmission from photoreceptors to first-order visual interneurons.

\section{Partial recovery of visual and mechanosensory behavior}

Visual behavior has been measured by a semiquantitative assay tracking flies in an illuminated arena with two black bars at opposite positions. Wild-type Drosophila will keep running back and forth between the two stripes for several hours (Götz, 1980). $h d c^{J K 910}$ behaves in this paradigm like wild type behaves in the dark, showing disoriented random walks. After histamine is fed to the mutant, partial recovery of wild-type behavior is observed (Fig. $4 a$, rescue). In our experiments all wild-type flies, none of the mutants, and 16 out of 22 histamine-fed mutants showed clearly oriented walks, demonstrating that these flies are able to detect visually the two stripes. To investigate the effects of histamine feeding on normal flies, we subjected wild type to the same histamine-diphosphate treatment. Interestingly, we observed a kind of general arousal effect in the histamine-fed wild types. These flies ran back and forth faster between the stripes, producing per unit time a larger number of straight tracks across the arena compared with that of the untreated wild-type group.

When a particular bristle on the thorax, the posterior scutellar macrochaeta, or the surface of the eye, which is covered with small interommatidial bristles, is gently touched using a mounted eyelash, wild-type flies will respond with $\sim 80 \%$ probability by attempting to escape or by scratching the touched region of the body surface with their legs. In $h d c^{J K 910}$ mutants, this response is reduced to $\sim 10-15 \%$, a value just above the background levels $(5 \%)$ that were determined in simulated stimulation of wild-type flies [stopping the eyelash just before it touches the bristle or the eye (cf. Melzig et al., 1996)]. After histamine is fed to the mutants, the response level of both reflexes is restored to $\sim 60 \%$ (Fig. 4b). Similarly, in a grooming assay, wild-type flies clean their body surface of Orange G dust almost completely (97\%) within a $4 \mathrm{hr}$ grooming time, whereas $h d c^{J K 910}$ mutants are much slower and barely reach 50\% (Melzig et al., 1996). Histamine-fed mutants do not achieve wild-type grooming efficiencies, but their performance is highly significantly improved compared with that of untreated mutants (Fig. 4c). 


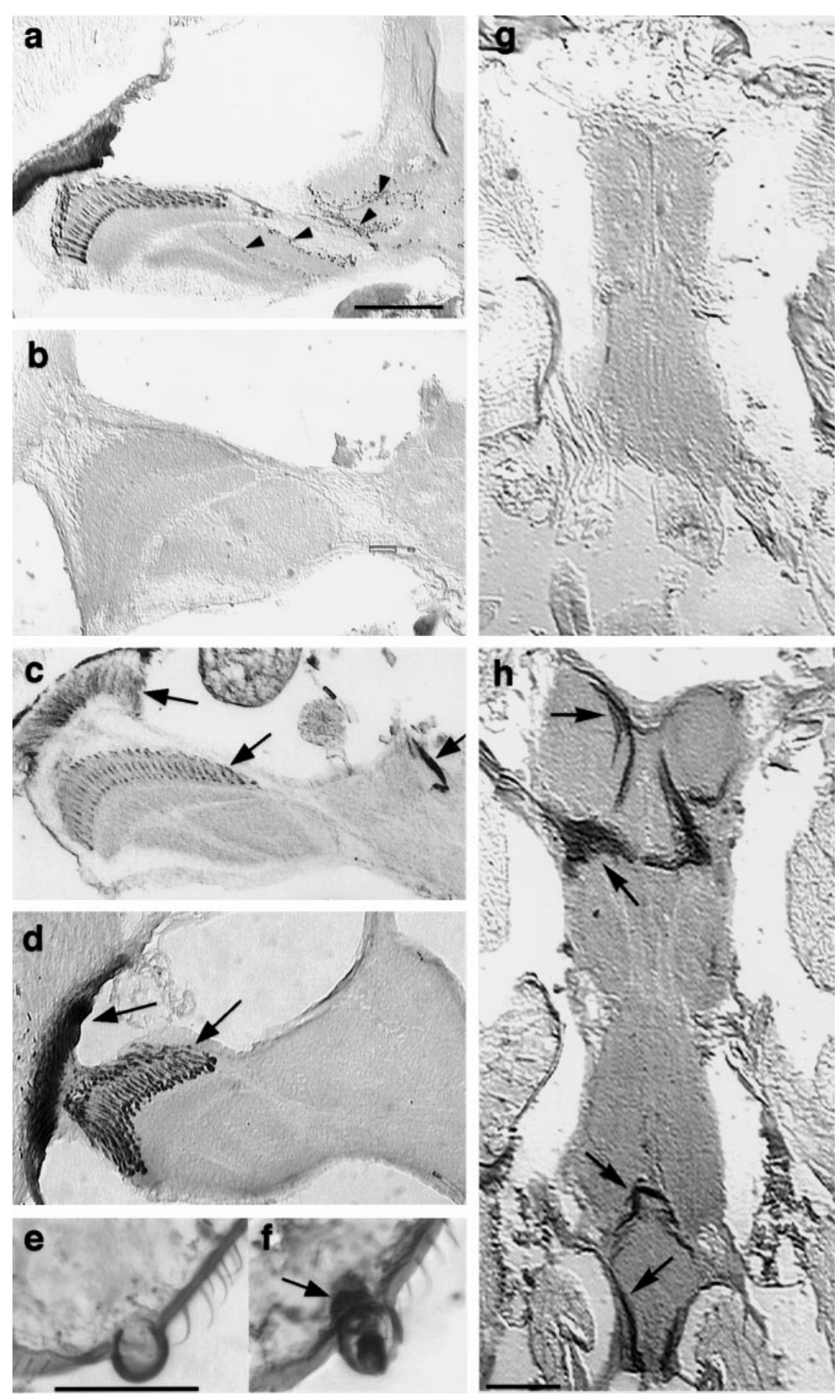

Figure 2. Immunohistochemical rescue of the $h d c^{J K 910}$ mutant. The staining localizes histamine in frozen sections of adult Drosophila. $a-d$, Left halfbrain of a wild-type fly $(a)$, an $h d c^{J K 910}$ mutant $(b)$, an $h d c^{J K 910}$ mutant after feeding on histaminediphosphate solution for $3 \mathrm{hr}(c)$, and a heat-shocked mutant that had been transformed by a heat-shock promoter- $h d c$-cDNA construct $(d)$. Note the strong staining in $c$ of the photoreceptor terminals R1-8 (long arrows) and the fibers of the antennal nerve (short arrow; compare with Fig. 1, left hemisphere). The preparation in $d$ demonstrates vigorous uptake of ubiquitously synthesized histamine into photoreceptors (arrows) but suggests that uptake into antennal mechanosensory neurons may require higher systemic concentrations or longer incubations (compare with text). The perikarya and processes of intrinsic neurons in lobula and central brain ( $a$, arrowheads) do not take up histamine even after long incubation $(c, d$; compare with $a$ and Fig. 1, right hemisphere). $e-h$, Corresponding phenomena observed for the mechanosensory bristle neurons $(e, f)$ and their projections in the thoracoabdominal ganglia $(g, h)$. Untreated $h d c^{J K 910}$ mutants $(e, g)$ are devoid of staining; after histamine feeding, the bases and sensory neurons of cuticular bristles $(f$, arrow $)$ and their axons and terminals in the ganglia $(h$, arrows) show strong histamine uptake (compare with Fig. 1, left hemisphere), whereas the intrinsic neurons remain unstained (Fig. 1, right hemisphere). Scale bars, $50 \mu \mathrm{m}$.

\section{DISCUSSION}

In the wild type, it must be assumed that all cells containing histamine selectively express the $h d c$ gene. This was shown by in situ hybridization for photoreceptors (Burg et al., 1993) and may be postulated for mechanoreceptors and intrinsic neurons because of the fact that the $h d c^{J K 910}$ mutation eliminates all immunohistochemical staining. The present results demonstrate, however, that the histaminergic system in Drosophila consists of two different subsystems.

Histamine-containing structures belonging to the peripheral nervous system (shown in Fig. 1, left hemisphere) possess an uptake mechanism that is specific for these cells and accumulates histamine against a high concentration gradient, as may be concluded from the large staining difference between receptor terminals and surrounding tissues in the immunohistochemical preparations of histamine-fed or transgenically rescued mutants. Our electrophysiological and behavioral tests show that this uptake mechanism is sufficient to primarily restore the function of these cells in the mutants from the low levels of histamine that are systemically provided by feeding histamine or by the ubiquitous expression of an $h d c$ transgene. Histamine uptake into photo- and mechanoreceptors is not restricted to flies with the allele $h d c^{J K 910}$ 

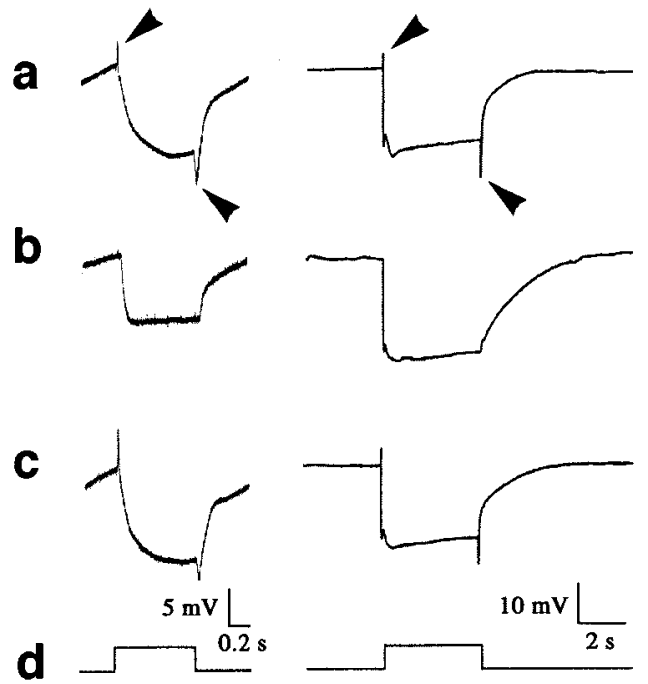

Figure 3. Rescue of synaptic transmission. $a-c$, Electroretinogram recordings of wild type $(a)$, untreated $h d c^{J K 910}$ mutants $(b)$, or mutants either fed on histamine-diphosphate solution $(c$, left $)$ or heat-shocked after transformation with a heat-shock $h d c$-cDNA construct $(c$, right) are shown. At light "on," wild-type ERGs show a positive "on-transient"; at light "off," a negative "off-transient" is shown ( $a$, arrowheads). Both transients are missing in the mutant $(b)$ but can be rescued by histamine feeding or ubiquitous expression of an $h d c$ transgene $(c) . d$, The durations and luminances of the light pulses were different in the left and right recordings (see Materials and Methods).

but is likewise found for the allele $h d c^{P 211}$ (data not shown) that consistently lacks histamine in the head (Melzig et al., 1996). The reasons why photoreceptors take up histamine more reliably than do mechanoreceptors are not known but may relate to the special property of sustained photoreceptor depolarization under photopic light conditions. This difference in histamine uptake between photo- and mechanoreceptors correlates, however, with the variability of mechanoreceptor staining observed in the mutants $h d c^{P 217}$ and $h d c^{P 218}$ (Melzig et al., 1996). Many factors may contribute to the fact that complex behavior in the rescued mutants is not fully restored, such as the possibility of miswiring of neuronal circuitry during development in the absence of histamine and specific or unspecific effects of unnatural concentrations of histamine in histaminergic or nonhistaminergic neurons or in the extracellular fluids.

The second subsystem is represented by the histaminecontaining neurons intrinsic to the CNS (shown in Fig. 1, right hemisphere). In the $h d c$ mutants selective staining of these neurons has never been observed. Because low concentrations of homogeneously distributed histamine are present in the CNS neuropil after histamine feeding or heat-shock of transgenic flies, as revealed by increased homogeneous "background" staining compared with that in untreated mutants, these cells apparently lack the histamine uptake mechanism that is responsible for the staining of receptor neurons. This interpretation depends on the assumption that in the mutants these cells still exist. Because in the wild type these cells can presently be identified only because of their histamine content, there is no way to prove this assumption. However, in view of the current understanding of developmental genetics, it seems extremely unlikely that two independent mutations in the $h d c$ gene could both cause a selective elimination of only these cells during neurogenesis.

The difference in histamine uptake between PNS and CNS a)
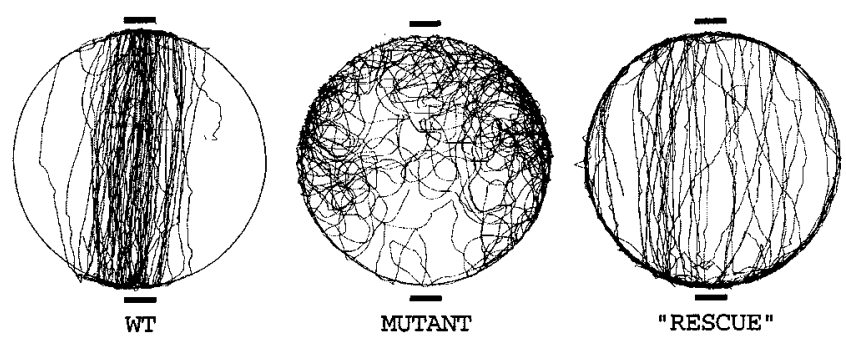

b)
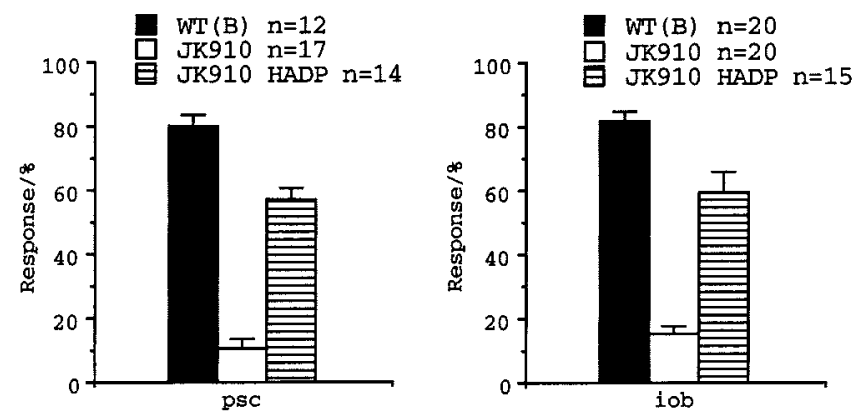

c)

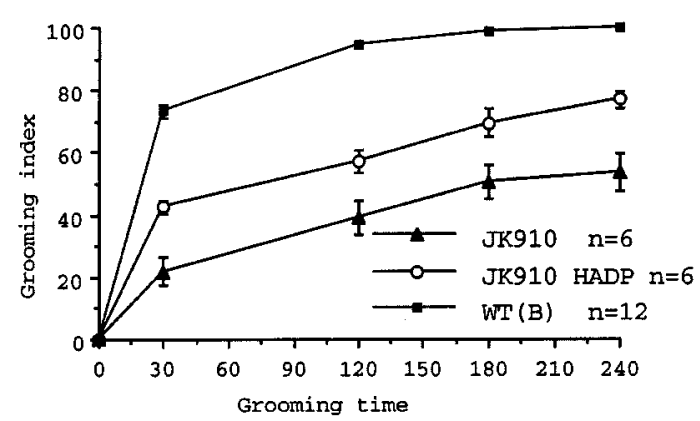

Figure 4. Behavioral rescue. $a$, Representative tracks of three individual flies in the arena of Buridan's paradigm are shown. Wild-type flies keep running back and forth between the two black stripes (horizontal bars); $h d c^{J K 910}$ mutants walk erratically with no preferred direction; after feeding on histamine-diphosphate solution (HADP), most of these mutants show near-normal tracks that cannot always be discriminated from wildtype tracks. $b$, Scratch or escape response probability after stimulation of the posterior scutellar macrochaeta ( $p s c)$ or the interommatidial bristles (iob) drops to near background levels in the $h d c^{J K 910}$ mutant but significantly recovers after the mutants are fed on histamine-diphosphate. $c$, Grooming behavior is dramatically impaired in $h d c^{J K 910}$ mutants ( filled triangles) but can be significantly improved in the mutant by histaminediphosphate feeding (open circles). The number of flies tested in each experiment $(n)$ is shown.

neurons is likely to have a functional correlate. In the PNS, histamine functions as a fast "classical" neurotransmitter with highly localized postsynaptic targets. For fly photoreceptors, this has been demonstrated conclusively by commonly accepted criteria, such as histamine presence, synthesis, and uptake (see below); electrophysiology and pharmacology of postsynaptic effects; and mutants (compare with the introductory remarks). The present rescue of the mutants demonstrates that histamine is not only necessary but also sufficient for both photoreceptor and mechanoreceptor function. In the CNS neurons, however, a role of histamine as a fast transmitter seems less likely because it 
presumably cannot be removed efficiently from the synaptic cleft. Thus we speculate that here histamine may instead act more diff usely and slowly, as would be expected for a neurohormone or neuromodulator for which fast removal from the release site is not required. A similar interpretation was given for the intrinsic histamine-immunoreactive cells in the thoracoabdominal ganglia of Drosophila and Calliphora purely on morphological grounds (Nässel et al., 1990). [Note that calypteran flies such as Musca or Calliphora contain histamine only in photoreceptors and intrinsic cells homologous to those seen in Drosophila but not in mechanosensory axons and terminals (Buchner et al., 1993).] In Aplysia, both fast and slow postsynaptic potentials were recorded when identifiable histamine-containing cerebral neurons were activated (McCaman and Weinreich, 1985). However, no information on histamine uptake of these neurons seems to be available.

Our results also suggest a less stringent hypothesis for the possible molecular interpretation of the two alleles $h d c^{P 217}$ and $h d c^{P 218}$ that lack histamine only in the intrinsic neurons. Previously, it has been speculated that in these alleles a specific regulatory sequence of the $h d c$ gene may be defective that is exclusively responsible for HDC expression in the intrinsic CNS neurons (Melzig et al., 1996). A less stringent hypothesis, suggested by our present results, is that any mutation destroying the cell specificity of HDC expression but still permitting low-level general expression could generate the observed phenotype. Histamine synthesized anywhere could diffuse to cells with specific uptake mechanisms and accumulate there to functional and immunohistochemically detectable concentrations.

Selective uptake of exogenously supplied biogenic amines into mutant neurons that normally synthesize the amine has been observed previously. The larval CNS of Drosophila mutants lacking the serotonin (5-HT) synthetic enzyme dopa decarboxylase (DDC) incubated in low concentrations of 5-HT shows 5-HT immunoreactivity (IR) similar to that of the wild type (Vallés and White, 1986). No uptake-incompetent neurons were described. In genetic mosaics, DDC-negative neurons displayed 5-HT IR even without exogenously supplied 5-HT, when their axons arborized in 5-HT-containing neuropils (Vallés, 1987; cited in Restifo and White, 1990). Because no physiological information on 5-HT function in Drosophila is presently available, these data neither support nor contradict the speculative proposal of a neuromodulatory role of histamine in those neurons that are uptakeincompetent under our conditions. A functional rescue, comparable with the behavioral aspects of the present results, was recently observed when adult mutants for the octopamine synthetic enzyme tyramine $\beta$-hydroxylase were fed on octopaminecontaining medium (Monastirioti et al., 1996).

No simple hypothesis can explain the immunohistochemical and behavioral effects of histamine feeding in wild-type flies. It is intriguing that a similar decrease in staining has been reported for serotonin immunoreactivity in flies heterozygous for a deficiency affecting $D d c$, the gene that codes for dopa decarboxylase, after incubation in relatively high concentrations of serotonin (Vallés, 1987; cited in Restifo and White, 1990). Feedback loops reducing synthesis or increasing release may cause such a phenomenon. Less specific effects like altered osmotic or $\mathrm{pH}$ conditions cannot be excluded, however. Similarly, the behavioral changes observed in the wild type after histamine feeding must be interpreted with great caution because imbibed histamine may possibly have various indirect effects on locomotor activity or the internal state of the fly. The described phenomena fit well, however, with the speculation of a modulatory function of histamine released from intrinsic CNS neurons. Careful behavioral analysis of the mutants and quantitative control of histamine concentrations in the hemolymph might help to clarify the question as to whether the intrinsic histaminergic neurons in the wild type are involved in the regulation of behavioral activity as suggested by the present observations.

One of the criteria for a substance to be accepted as a classical neurotransmitter is the demonstration of a mechanism for its removal from the synaptic cleft. This can be achieved by enzymatic degradation, as has long been known for acetylcholine. Little information is available on the metabolic degradation of histamine in insects. In thoracic ganglia of cockroaches and crickets, histamine can be acetylated to $\mathrm{N}$-acetylhistamine (Huggins and Woodruff, 1968; Elias and Evans, 1983). In Drosophila, only a small fraction of the radioactivity detected in head tissues after incubation in $20 \mu \mathrm{M}\left[{ }^{3} \mathrm{H}\right]$ histamine was associated with $N$-acetylhistamine and imidazole-4 acetic acid (Sarthy, 1991). This indicates a slow breakdown process that nonetheless may represent a functional component of the histamine inactivation mechanism, analogous to the $O$-methylation of the catecholamine neurotransmitters. An alternative possibility to terminate transmitter action is represented by high-affinity uptake of the substance into the presynaptic neuron or surrounding glia cells. This mechanism has been demonstrated for most classical transmitters, but in mammals histamine uptake appears to be restricted to glia and may be lacking in histaminergic neurons (Smits et al., 1988; Schwartz et al., 1991; Huszti et al., 1998). Because it seems highly unlikely that the histamine uptake observed here in all histaminecontaining PNS neurons, namely, compound eye photoreceptors, ocellar photoreceptors (data not shown), subretinal photoreceptors (Hofbauer and Buchner, 1989) (data not shown), and bristle mechanoreceptors (but not campaniform or scolopidial sensilla), could have an artifactual cause that is unrelated to histamine function in these cells, we propose that our experiments localize a specific mechanism that transports histamine against a concentration gradient across the plasma membrane. Selective $\left[{ }^{3} \mathrm{H}\right]$ histamine uptake that is driven by the $\mathrm{Na}^{+}$gradient has been described recently for an arthropod photoreceptor (Stuart et al., 1996). Our present results demonstrate that the proper function of a presumably homologous mechanism in Drosophila can be assayed in behavioral, electrophysiological (ERG), or immunohistochemical experiments. These techniques may now be used for screening mutants induced on a $h d c^{J K 910}$ background for a defective uptake mechanism and may thus provide the basis for identifying and cloning the corresponding genes.

\section{REFERENCES}

Buchner E, Buchner S, Burg MG, Hofbauer A, Pak WL, Pollack I (1993) Histamine is a major mechanosensory neurotransmitter candidate in Drosophila melanogaster. Cell Tissue Res 273:119-125.

Burg MG, Sarthy PV, Koliantz G, Pak WL (1993) Genetic and molecular identification of a Drosophila histidine decarboxylase gene required in photoreceptor transmitter synthesis. EMBO J 12:911-919.

Coombe PE (1986) The large monopolar cells L1 and L2 are responsible for ERG transients in Drosophila. J Comp Physiol [A] 159:655-666.

Elias MS, Evans PD (1983) Histamine in the insect nervous system: distribution, synthesis and metabolism. J Neurochem 41:562-568.

Götz KG (1980) Visual guidance in Drosophila. In: Development and neurobiology of Drosophila (Siddiqi O, Babu P, Hall LM, eds), pp 391-407. New York: Plenum.

Gruhn M (1995) Elektrophysiologische Charakterisierung der Fliegenstämme $c s p^{K 16}$ und $c s p^{\chi^{16}}$ von Drosophila melanogaster. Master's thesis, University of Würzburg. 
Hardie RC (1989) A histamine-activated chloride channel involved in neurotransmission at a photoreceptor synapse. Nature 339:704-706.

Heisenberg M (1971) Separation of receptor and lamina potentials in the electroretinogram of normal and mutant Drosophila. J Exp Biol 55:85-100.

Hofbauer A, Buchner E (1989) Does Drosophila have seven eyes? Naturwissenschaften 76:335-336.

Huggins AK, Woodruff GN (1968) Histamine metabolism in invertebrates. Comp Biochem Physiol 26:1107-1111.

Huszti Z, Prast H, Tran MH, Fischer H, Philippu A (1998) Glial cells participate in histamine inactivation in vivo. Naunyn Schmiedebergs Arch Pharmacol 357:49-53.

McCaman RE, Weinreich D (1985) Histaminergic synaptic transmission in the cerebral ganglion of Aplysia. J Neurophysiol 53:1016-1037.

Melzig J, Buchner S, Wiebel F, Wolf R, Burg M, Pak WL, Buchner E (1996) Genetic depletion of histamine from the nervous system of Drosophila eliminates specific visual and mechanosensory behavior. J Comp Physiol [A] 179:763-773.

Monastirioti M, Linn Jr CE, White K (1996) Characterization of Drosophila tyramine $\beta$-hydroxylase gene and isolation of mutant flies lacking octopamine. J Neurosci 16:3900-3911.

Nässel DR, Elekes K (1992) Aminergic neurons in the brain of blowflies and Drosophila: dopamine- and tyrosine hydroxylase-immunoreactive neurons and their relationship with putative histaminergic neurons. Cell Tissue Res 267:147-167.

Nässel DR, Pirvola U, Panula P (1990) Histaminelike immunoreactive neurons innervating putative neurohaemal areas and central neuropil in the thoraco-abdominal ganglia of the flies Drosophila and Calliphora. J Comp Neurol 297:525-536.

Pak WL (1975) Mutants affecting the vision of Drosophila melanogaster.
In: Handbook of genetics (King RC, ed), pp 703-733. New York: Plenum.

Pollack I, Hofbauer A (1991) Histamine-like immunoreactivity in the visual system and brain of Drosophila melanogaster. Cell Tissue Res 266:391-398.

Restifo LL, White K (1990) Molecular and genetic approaches to neurotransmitters and neuromodulator systems in Drosophila. Adv Ins Physiol 22:115-219.

Sarthy PV (1991) Histamine: a neurotransmitter candidate for Drosophila photoreceptors. J Neurochem 57:1757-1768.

Schwartz JC, Arrang JM, Garbarg M, Pollard H, Ruat M (1991) Histaminergic transmission in the mammalian brain. Physiol Rev 71:1-51.

Skingsley DR, Laughlin SB, Hardie RC (1995) Properties of histamineactivated chloride channels in the large monopolar cells of the dipteran compound eye: a comparative study. J Comp Physiol [A] 176:611-623.

Smits RPJM, Steinbusch HWM, Mulder AH (1988) Studies on the specificity of uptake and release of radiolabelled histamine in rat brain slices. Neurochem Int 12:193-202.

Stuart AE, Morgan JR, Mekeel HE, Kempter E, Callaway JC (1996) Selective, activity-dependent uptake of histamine into an arthropod photoreceptor. J Neurosci 16:3178-3188.

Vallés AM (1987) Serotonin-containing neurons in Drosophila melanogaster, a neurogenetic analysis. PhD thesis, Brandeis University, Waltham MA.

Vallés AM, White K (1986) Development of serotonin-containing neurons in Drosophila mutants unable to synthesize serotonin. J Neurosci 6:1482-1491.

Vandervorst P, Ghysen A (1980) Genetic control of sensory connections in Drosophila. Nature 286:65-67. 